\title{
Sex-specific association between fibroblast growth factor 21 and type 2 diabetes: a nested case-control study in Singapore Chinese men and women
}

\author{
Yeli Wang ${ }^{1}$, Woon-Puay Koh ${ }^{1,2}$, Jian-Min Yuan ${ }^{3,4}$ and An Pan ${ }^{5^{*}}$
}

\begin{abstract}
Background: Fibroblast growth factor 21 (FGF-21) is mainly secreted by liver and has been reported to be involved in the pathogenesis of type 2 diabetes. Some prospective studies have shown a positive association between FGF21 and diabetes risk. However, no study has examined whether the association differed by sex, which has been reported between FGF-21 and atherosclerosis. Therefore, we prospectively evaluated the sex-specific association between FGF-21 and diabetes in a Chinese population.

Methods: Serum FGF-21 concentration was measured in a case-control study comprising of 251 incident diabetes cases and 251 age-sex-matched controls nested within a prospective population-based cohort, the Singapore Chinese Health Study. At blood collection between 1999 and 2004, participants were free of diagnosed diabetes, cardiovascular disease, and cancer. Incident self-reported diabetes cases were identified at follow-up II interview (2006-2010). Odds ratio (OR) and 95\% confidence interval (Cl) were calculated using multivariable logistic regression models.

Results: After adjustment for risk biomarkers of diabetes including lipids, liver enzymes and inflammatory marker, the OR of type 2 diabetes with per one unit increment in log FGF-21 concentration was 1.16 (95\% Cl 0.90-1.50). Significant interaction was found with sex (P-interaction $=0.029)$ : the $\mathrm{OR}(95 \% \mathrm{Cl})$ was $1.50(1.00-2.25)$ in women and $0.89(0.52-1.53)$ in men.

Conclusions: Higher serum FGF-21 level was associated with an increased risk of diabetes in Chinese women but not in men. The sex difference in the association between FGF-21 and diabetes risk deserves further investigation and replication in other populations.
\end{abstract}

Keywords: Fibroblast growth factor 21, Nested case-control study, Type 2 diabetes, Prospective study

\section{Background}

Fibroblast growth factor 21 (FGF-21) is a hormone secreted mainly by the liver, as well as by adipose tissue, pancreas, and skeletal muscle [1]. FGF-21 has increasingly attracted attention recently due to its potential beneficial role in glucose homeostasis and lipid metabolism [2]. Animal studies have shown that FGF-21 ameliorates

\footnotetext{
* Correspondence: panan@hust.edu.cn

${ }^{5}$ Department of Epidemiology and Biostatistics, Ministry of Education Key Laboratory of Environment and Health and State Key Laboratory of Environmental Health (incubation), School of Public Health, Tongji Medical College, Huazhong University of Science and Technology, Wuhan, Hubei Province 430030, China

Full list of author information is available at the end of the article
}

hyperglycemia, hyperlipidemia, and insulin resistance [3], and may thus prevent the development of type 2 diabetes.

Despite the favorable metabolic changes observed in animal studies, cross-sectional studies in humans have found that circulating FGF-21 levels were paradoxically elevated with conditions of metabolic dysregulations such as metabolic syndrome [4] and type 2 diabetes [5], although a temporal relationship cannot be determined from these studies. So far, only three prospective cohort studies with relatively small cases numbers (patients with diabetes ranged from 54 to 123) have been conducted, and all reported a positive association between higher FGF-21 levels and increased risk of type 2 diabetes [6-8]. 
However, it is largely unknown whether nonalcoholic fatty liver disease (NAFLD), a condition associated with both diabetes and FGF-21 levels [9], may explain or modify the association between FGF-21 and diabetes risk. Moreover, recent studies have reported an interaction between FGF21 and sex in its association with atherosclerosis and bone mineral density [10-12], with an association observed in women but not in men, but this has not been studied in the context of diabetes. Furthermore, two studies have examined whether FGF-21 added substantial value in predicting diabetes risk, and the results were inconsistent $[6,8]$. While one study reported that the predictive utility of FGF-21 was as good as the oral glucose tolerance test (OGTT) [8], the other study found that including FGF-21 did not improve diabetes prediction [6].

In this nested case-control study within the Singapore Chinese Health Study, we studied the association between FGF-21 and risk of type 2 diabetes with adjustment of established diabetes risk factors including two liver enzymes alanine transaminase (ALT) and gammaglutamyltransferase (GGT). ALT and GGT could serve as surrogate markers of NAFLD [13], and we have previously reported a positive association between the two liver enzymes with diabetes risk [14]. We also performed stratified analysis to explore the potential heterogeneity among different subgroups, particularly sex. Additionally, we assessed the incremental value of FGF-21 in diabetes risk prediction over established risk factors in this population.

\section{Methods}

\section{Study population}

The design of the Singapore Chinese Health Study has been described in detail previously [15]. Briefly, the prospective cohort was established between 1993 and 1998, and recruited 63,257 Chinese adults aged between 45 and 74 years. Baseline information on diet, lifestyle habits and medical history was collected at recruitment by an in-person interview. Between 1999 and 2004, follow-up I interviews were conducted via telephone. Among 52,322 participants who were re-contacted successfully, a total of 32,535 individuals donated their bio-specimens. Between 2006 and 2010, follow-up II interviews were conducted via telephone, and a total of 39,528 participants were re-contacted successfully. The study protocol was approved by the Institutional Review Boards at the National University of Singapore and the University of Pittsburgh. Informed consent was provided and completed by each participant at the baseline interview.

\section{Ascertainment of diabetes and other covariates}

At baseline and both follow-up interviews, history of physician-diagnosed diabetes was asked by the question: "Have you been told by a doctor that you have diabetes?"
If the participant answered "yes", he or she was also asked for the age of first diagnosis. The robustness and accuracy of the self-reported diabetes data has been confirmed in a validation study [16].

Height and body weight were self-reported at baseline and both follow-ups. Body mass index (BMI) was calculated as weight (kilograms) divided by height (meters) squared. For those participants with missing height or weight, BMI was calculated using imputed data derived from the linear regression equation: Weight $=y$-intercept + gradient $\times$ height. The values for the $y$-intercept and gradient were derived from gender-specific weight-height regression lines, which were obtained from all cohort participants with reported heights and weights.

\section{Establishment of nested case-control study}

For the current analysis, we established a nested case-control study of 251 incident cases and 251 matched controls within this cohort. All cases and controls were free of physiciandiagnosed diabetes, cardiovascular disease and cancer at baseline interview as well as the time of blood collection during 1999 and 2004. Among 571 participants who subsequently reported to be diagnosed with diabetes during follow-up II visit (2006-2010), we selected 292 cases who had hemoglobin $\mathrm{A} 1 \mathrm{c}\left(\mathrm{HbA}_{1 \mathrm{c}}\right)$ levels $<6.5 \%$ at blood donation to exclude undiagnosed diabetes. Controls were chosen from the remaining participants who did not develop diabetes or cardiovascular disease at follow-up II, and were matched for age ( \pm 3 years), date of blood collection ( \pm 6 months), sex (men, women), and dialect group (Cantonese, Hokkien) with the cases on a 1:1 ratio. Furthermore, controls were considered eligible if their baseline $\mathrm{HbA}_{1 \mathrm{c}}$ levels were less than $6.0 \%$. Some participants with insufficient serum samples ( $n=37$ ) or extreme FGF-21 levels (>3 standard deviation [SD], $n=4$ ) were excluded, leaving a total of 251 casecontrol pairs for the present study. The flowchart of the study design is shown in Additional file 1: Figure S1.

\section{Laboratory procedures}

Twenty-mL random morning blood was collected from each consenting participant and separated into plasma, serum, red blood cells, and buffy coat, and stored in $-80{ }^{\circ} \mathrm{C}$ freezers. Serum concentrations of FGF-21 and plasma levels of adiponectin were measured by ELISA/Evolis (Bio-Rad Laboratories, Hercules, CA). Plasma concentrations of high-sensitivity $\mathrm{C}$-reactive protein (hs-CRP), total cholesterol (TC), triglycerides (TG), high-density lipoprotein cholesterol (HDL-C), ALT and serum levels of GGT were measured via colorimetric method on a chemistry analyzer (AU5800 Analyzer, Beckman Coulter, Brea, CA). HbA levels in red blood cells were measured by HPLC method using Bio-Rad Variant II ${ }^{\mathrm{Tm}}$ System (Bio-Rad Laboratories, Hercules, CA). 


\section{Statistical analysis}

Because of the different distributions between men and women, baseline characteristics were presented for men and women separately. For prospective analyses, study participants were divided into quartiles according to the sex-specific distribution of FGF-21 levels among control participants, and the lowest quartile served as the reference group. Conditional logistic regression models were used to calculate the odds ratio (OR) and corresponding 95\% confidence interval (CI) between FGF-21 and diabetes. Model 1 was adjusted for age (continuous), smoking (never, ever smoker), alcohol intake (never, ever drinker), weekly moderate-to-vigorous physical activity $(<0.5, \geq 0.5 \mathrm{~h} /$ week $)$, education level (primary school and below, secondary or above), history of hypertension (yes, no), fasting status (yes, no) and BMI (continuous). In addition, since FGF-21 levels were associated with hs-CRP levels [17], dyslipidemia [3], and nonalcoholic fatty liver disease [17], we further adjusted for the metabolic biomarkers (hs-CRP, TG, HDL-C, GGT, and ALT) both in quartiles and as continuous variables to examine their impact on the association between FGF-21 and diabetes. Because of the significant interaction with sex, we repeated the abovementioned analysis in men and women separately, and we have additionally adjusted for menopausal status in women. We then used restricted cubic spline regression with 4 knots at 5\%, 35\%, 65\% and 95\% percentiles of original value of FGF-21 to examine a possible non-linear relation between FGF-21 and diabetes risk. When the nonlinear hypothesis was rejected, we also calculated the diabetes risk associated with per 1 unit increment in log FGF-21 levels, in order to compare our results with previous studies $[6,7]$. Moreover, we tested potential interactions with age ( $<60$ or $\geq 60$ years), sex (men, women), fasting status (yes, no), BMI $\left(<23\right.$ or $\left.\geq 23 \mathrm{~kg} / \mathrm{m}^{2}\right)$, physical activity $(<0.5$ or $\geq 0.5 \mathrm{~h} /$ week), plasma levels of hs-CRP, GGT, ALT, TG, or HDL-C (above or below median levels of each biomarker) by adding an interaction term (each binary variable $\times \log$-transformed FGF-21) to the regression models in the men and women separately. Potential interaction with menopausal status was additionally tested in women. In the stratified analysis by non-matching variables, unconditional logistic regression models were used with additional adjustment for sex and dialect group (Cantonese, Hokkien).

The predictive utility of FGF-21 for diabetes prediction was subsequently examined. The optimal cutoff value was derived by using receiver-operating characteristic (ROC) analysis and Youden index [18]. Base model 1 included age and BMI; base model 2 additionally included smoking status, history of hypertension, and levels of TG, HDL-C, and random glucose. In addition, we built the base model 3 to further include adiponectin and hsCRP. The improvement in discrimination between the parsimonious model and the model plus binary FGF-21 was examined by comparing area under receiveroperating characteristic curve (AUC) using DeLong's method [19]. Moreover, we used the category-free net reclassification improvement (NRI) and integrated discrimination improvement (IDI) statistics recommended by Pencina et al. [20, 21]. Furthermore, we used Akaike information criteria (AIC) to assess the goodness-of-fit of all models, where lower AICs indicate better model fit. Analyses were performed with Stata software, version 14.0 (Stata Corp, College Station, Texas). Two-sided $P$ values $<0.05$ were considered to be statistically significant.

\section{Results}

Among the cases, the mean age of diagnosis of incident type 2 diabetes (SD) was 63.2 (6.4) years and the mean duration (SD) between blood donation and diagnosis was 4.0 (1.7) years. The sex-specific baseline characteristics of cases and controls stratified by sex are shown in Table 1. In both men and women, cases had higher BMI and were more likely to have hypertension compared to matched controls. No significant differences were found for education levels, smoking status and alcohol consumption. In addition, 54 women (20\%) were premenopausal and 214 women $(80 \%)$ were at postmenopausal status. For blood biomarkers in both men and women, cases had higher levels of FGF-21, $\mathrm{HbA}_{1 \mathrm{c}}$, hs-CRP, TG, GGT, ALT, but lower levels of adiponectin and HDL-C. Among both cases and controls, serum FGF-21 levels were positively correlated with ALT, GGT, TG and hsCRP, and negatively correlated with HDL-C and adiponectin (Additional file 1: Table S1). Similar pattern was found in men and women (data not shown).

The association between FGF-21 and risk of type 2 diabetes is presented in Table 2 . In the total study population, higher levels of serum FGF-21 were significantly associated with increased diabetes risk after adjustment for age, sex, lifestyle factors, fasting status and BMI; the OR $(95 \%$ CI) comparing the highest versus lowest quartile was 2.70 (1.33-5.50; $P$-trend $=0.015)$. However, after mutual adjustment for quartiles of hs-CRP, TG, HDL-C, GGT, and ALT, the association became statistically non-significant in model 2 (OR 1.75; 95\% CI 0.76-4.01; $P$-trend $=0.23$ ). Significant interaction was found with sex ( $P$-interaction $=0.029)$, and the association was evident in women only (OR 4.19; 95\% CI 1.07-16.5; $P$-trend $=0.036$ ) but not in men (OR 1.20; 95\% CI $0.24-5.94 ; P$-trend $=0.70$ ) comparing the extreme quartiles of FGF-21 levels in model 2. In addition, cubic spline regression model suggested a linear relationship between FGF-21 and T2D risk in both men $(P$ for nonlinearity $=0.86)$ and women $(P$ for nonlinearity $=0.09$ ) (Fig. 1). Given the linear association, we further estimated for the every $1-\log \mathrm{pg} / \mathrm{mL}$ increment in FGF-21 levels, the ORs (95\% CIs) for diabetes in model 2 were 1.16 $(0.90-1.50)$ in the total study samples, $0.89(0.52-1.53)$ in 
Table 1 Baseline characteristics and liver enzymes of diabetes cases and matched controls in men and women, the Singapore Chinese Health Study ${ }^{a}$

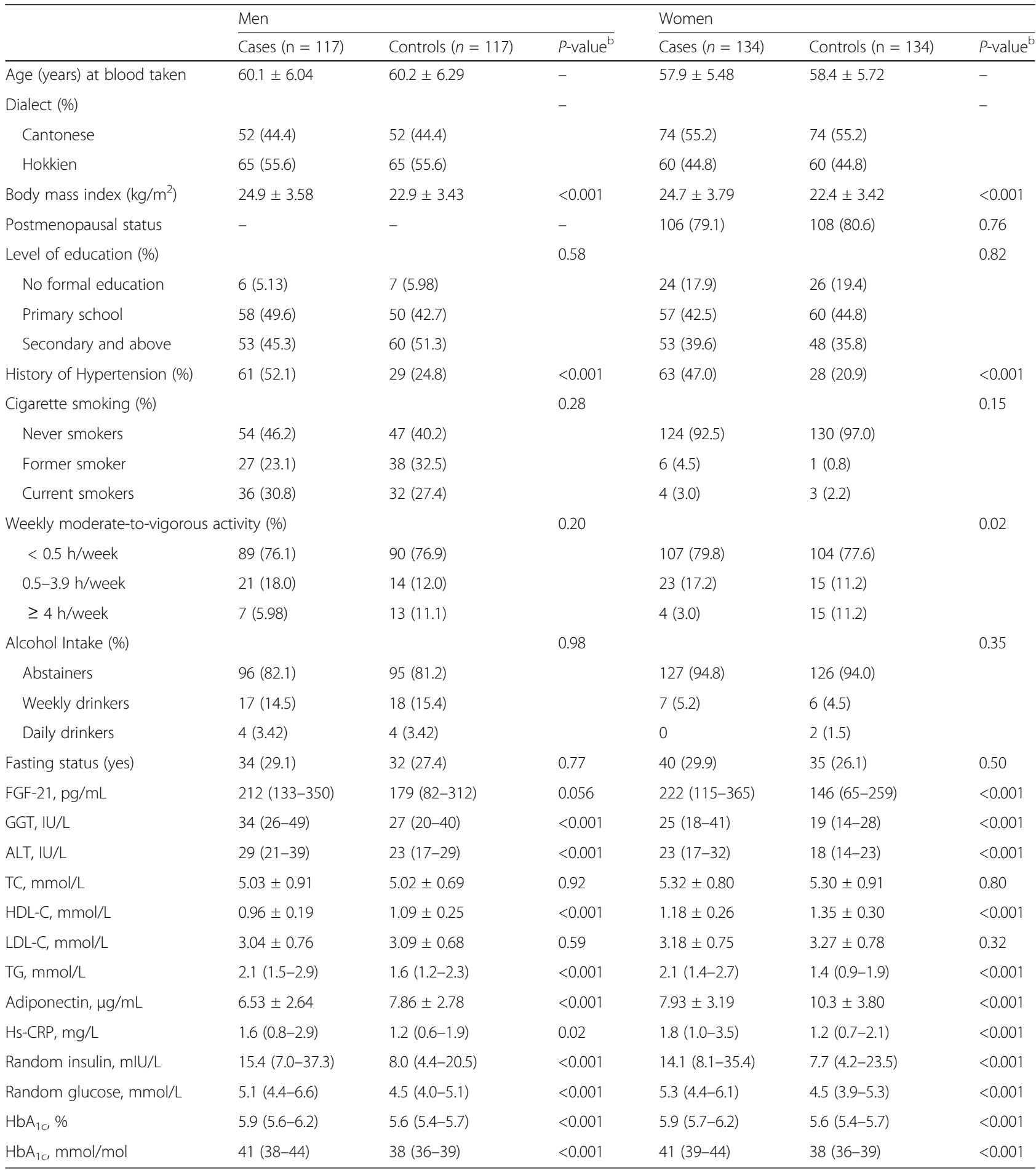

${ }^{a}$ Data are expressed as mean \pm standard deviation for continuous variables (normally distributed) and median (interquartile range) for continuous variables (skewed distributed), and $\mathrm{n}$ (percentage) for categorical variables. Cases and controls are matched on age at blood taken ( \pm 3 years), gender, dialect, and date of blood collection ( \pm 6 months)

${ }^{\mathrm{b}} \mathrm{P}$ values based on the chi-square test for categorical variables, student's t-test and Mann-Whitney test for continuous variable 
Table 2 Odds ratios (95\% confidence intervals) of type 2 diabetes associated with different levels of FGF-21 in men and women, the Singapore Chinese Health Study

\begin{tabular}{|c|c|c|c|c|c|c|}
\hline \multirow[t]{2}{*}{ Variables } & \multicolumn{4}{|c|}{ Quartiles of FGF-21 } & \multirow{2}{*}{$\begin{array}{l}P \text { for } \\
\text { trend }^{a}\end{array}$} & \multirow{2}{*}{$\begin{array}{l}\text { Per } 1 \text { log } \\
\text { increment }\end{array}$} \\
\hline & $\overline{\mathrm{Q} 1}$ & Q2 & Q3 & Q4 & & \\
\hline \multicolumn{7}{|l|}{ Whole dataset } \\
\hline Median (range) & $47(7-75)$ & $116(76-157)$ & $212(158-288)$ & $411(289-1607)$ & & \\
\hline Cases/controls & $31 / 64$ & $61 / 63$ & $64 / 62$ & $95 / 62$ & & \\
\hline Model $1^{\mathrm{b}}$ & 1.00 & $1.96(1.00-3.85)$ & $1.77(0.94-3.33)$ & $2.70(1.33-5.50)$ & 0.015 & $1.28(1.03-1.59)$ \\
\hline Model $1^{\mathrm{b}}+$ hs-CRP & 1.00 & $1.73(0.87-3.44)$ & $1.69(0.89-3.21)$ & $2.56(1.24-5.31)$ & 0.018 & $1.27(1.01-1.58)$ \\
\hline Model $1^{\mathrm{b}}+\mathrm{TG}, \mathrm{HDL}-\mathrm{C}$ & 1.00 & $1.76(0.85-3.67)$ & $1.47(0.74-2.94)$ & $1.91(0.87-4.20)$ & 0.20 & $1.19(0.93-1.52)$ \\
\hline Model $1^{b}+A L T, G G T$ & 1.00 & $1.79(0.88-3.63)$ & $1.77(0.90-3.49)$ & $2.10(0.98-4.50)$ & 0.09 & $1.20(0.95-1.51)$ \\
\hline Model $2^{c}$ & 1.00 & $1.53(0.70-3.32)$ & $1.49(0.72-3.10)$ & $1.75(0.76-4.01)$ & 0.23 & $1.16(0.90-1.50)$ \\
\hline \multicolumn{7}{|l|}{ Men } \\
\hline Median (range) & $59(7-82)$ & $133(83-179)$ & $240(180-312)$ & $483(317-1460)$ & & \\
\hline Cases/controls & $16 / 30$ & $32 / 29$ & $32 / 29$ & $37 / 29$ & & \\
\hline Model $1^{\mathrm{b}}$ & 1.00 & $2.00(0.76-5.28)$ & $1.43(0.55-3.70)$ & $1.66(0.53-5.21)$ & 0.68 & $0.95(0.65-1.38)$ \\
\hline Model $1^{b}+$ hs-CRP & 1.00 & $1.80(0.65-4.97)$ & $1.35(0.51-3.55)$ & $1.71(0.52-5.65)$ & 0.63 & $0.95(0.64-1.41)$ \\
\hline Model $1^{\mathrm{b}}+\mathrm{TG}, \mathrm{HDL}-\mathrm{C}$ & 1.00 & $1.44(0.50-4.17)$ & $1.33(0.47-3.83)$ & $1.21(0.33-4.39)$ & 0.88 & $0.94(0.60-1.46)$ \\
\hline Model $1^{\mathrm{b}}+\mathrm{ALT}, \mathrm{GGT}$ & 1.00 & $1.66(0.56-4.98)$ & $1.59(0.54-4.69)$ & $1.27(0.33-4.80)$ & 0.81 & $0.85(0.55-1.31)$ \\
\hline Model $2^{c}$ & 1.00 & $1.32(0.36-4.80)$ & $1.67(0.47-5.95)$ & $1.20(0.24-5.94)$ & 0.70 & $0.89(0.52-1.53)^{\mathrm{e}}$ \\
\hline \multicolumn{7}{|l|}{ Women } \\
\hline Median (range) & $30(7-65)$ & $107(65-146)$ & $197(147-259)$ & $375(260-1607)$ & & \\
\hline Cases/controls & $15 / 34$ & $29 / 34$ & $32 / 33$ & $58 / 33$ & & \\
\hline Model $1^{b}$ & 1.00 & $2.13(0.76-5.97)$ & $2.15(0.87-5.28)$ & $4.73(1.69-13.2)$ & 0.004 & $1.60(1.16-2.19)$ \\
\hline Model $1^{b}+$ hs-CRP & 1.00 & $1.74(0.60-5.03)$ & $2.11(0.84-5.33)$ & $4.07(1.42-11.7)$ & 0.006 & $1.56(1.13-2.15)$ \\
\hline Model $1^{\mathrm{b}}+\mathrm{TG}, \mathrm{HDL}-\mathrm{C}$ & 1.00 & $2.28(0.67-7.74)$ & $1.87(0.67-5.20)$ & $4.37(1.31-14.6)$ & 0.033 & $1.49(1.02-2.17)$ \\
\hline Model $1^{\mathrm{b}}+\mathrm{ALT}, \mathrm{GGT}$ & 1.00 & $2.25(0.72-7.03)$ & $2.28(0.86-6.05)$ & $4.30(1.38-13.4)$ & 0.014 & $1.54(1.10-2.16)$ \\
\hline Model $2^{c}$ & 1.00 & $1.90(0.50-7.26)$ & $2.28(0.73-7.14)$ & $4.19(1.07-16.5)$ & 0.036 & $1.53(1.02-2.29)^{\mathrm{e}}$ \\
\hline Model $3^{d}$ & 1.00 & $1.81(0.46-7.14)$ & $2.27(0.72-7.14)$ & $3.88(0.99-15.3)$ & 0.047 & $1.50(1.00-2.25)$ \\
\hline
\end{tabular}

aLinear trend was tested by using the median level of each quartile of FGF-21

${ }^{\mathrm{b}}$ Model 1: adjusted for age at blood taken (continuous), smoking (never, ever smoker), alcohol intake (never, ever drinker), weekly moderate-to-vigorous activity $(<0.5, \geq 0.5$ hours/week), education level (primary school and below, secondary or above), history of hypertension (yes, no), fasting status (yes, no), and body mass index (continuous)

'Model 2: Model 1 plus hs-CRP (mg/L), TG (mmol/L), HDL-C (mmol/L), GGT (IU/L) and ALT (IU/L) (all in quartiles)

dMode 3: Model 2 plus menopausal status (premenopausal, postmenopausal status)

${ }^{\mathrm{e}}$ The $P$-interaction $=0.029$ between FGF- 21 and sex associated with incident type 2 diabetes risk

men and $1.53(1.02-2.29)$ in women. In a sensitivity analysis, we used continuous values of blood biomarkers instead of quartiles in the multivariable adjustment, and the results were materially changed: the ORs (95\% CIs) for diabetes with per-log increment in FGF-21 levels were 1.15 $(0.91-1.45)$ in the total population, $0.80(0.53-1.23)$ in men and $1.43(1.00-2.05)$ in women (data not shown). In addition, further adjustment for menopausal status in women had little impact on the association between FGF21 and diabetes (OR per log FGF-21: 1.50; 95\% CI 1.002.25). Moreover, no statistically significant interactions were found with other variables in the total study sample (Table 3), men and women (Table 4); however, the sample size in the stratified analyses was much smaller.
Since we only found a significant association among women, we limited the analysis for the predictive utility of FGF-21 to women only. The best cutoff predictive value for risk of diabetes was $123 \mathrm{pg} / \mathrm{mL}$ using Youden index in the ROC analysis. The sensitivity and specificity of the cutoff point were $75.7 \%$ and $41.4 \%$, respectively. The predictive performance of FGF-21 is presented in Table 5 and Additional file 1: Table S2. In all base models, addition of binary FGF-21 did not significantly improve the AUC (AUC changes range from 0.004 to $0.018, P$ values range from 0.11 to 0.56 ). However, adding binary FGF-21 resulted in statistically significant NRI (NRIs range from 0.358 to 0.388 , all $P<0.01$ ) and IDI (IDIs range from 0.013 to 0.028 , $P$ values range from 0.009 to 0.052 ) in all 3 models. 


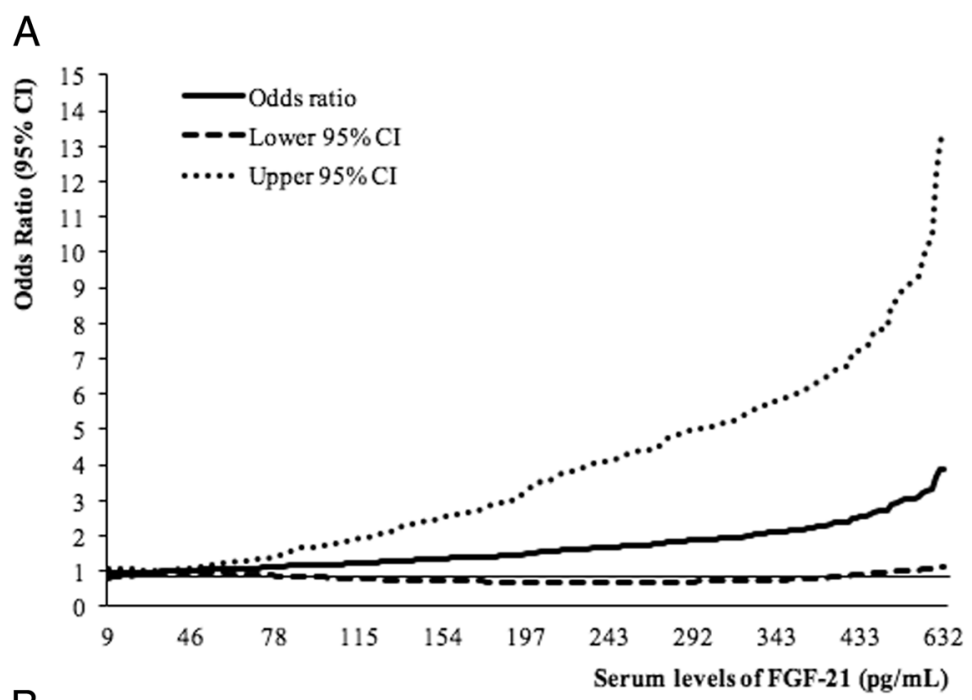

B

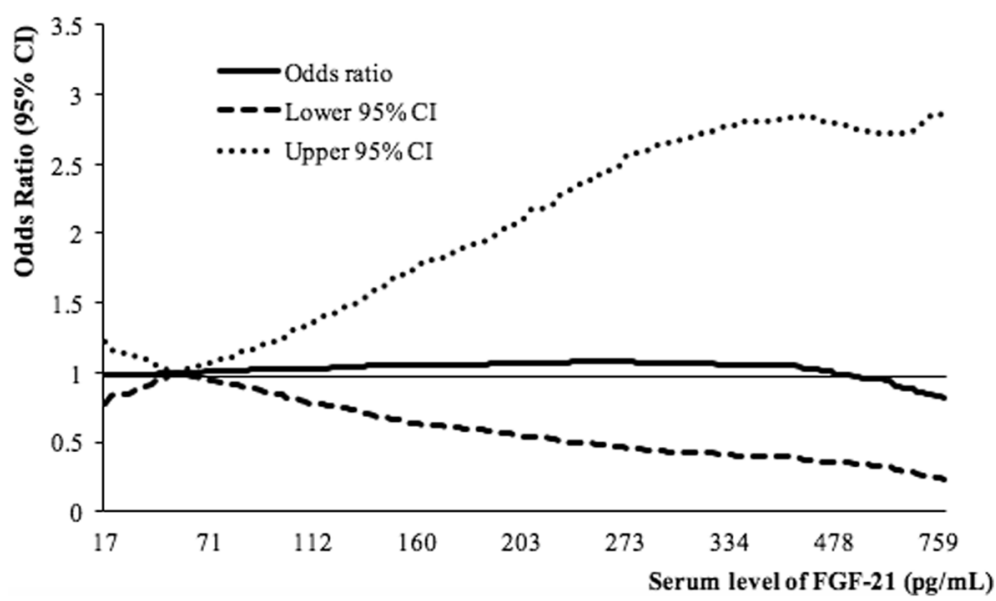

Fig. 1 Spline analysis of the association between serum levels of FGF-21 and incident type 2 diabetes in women (a) and men (b). The Solid line represents point estimates of relative risk for the association between FGF-21 and incident type 2 diabetes, and the dotted lines represent the upper and lower bound of $95 \% \mathrm{Cl}$. Study participants with the lowest and highest 1\% of FGF-21 were excluded to minimize the potential impact of outliers. Cubic spline analysis was used to examine the association between FGF-21 concentrations and risk of developing type 2 diabetes using the most fully-adjusted models from reported studies. $P$ for nonlinearity $=0.09$ in women (a) $P$ for nonlinearity $=0.86$ in men (b) in the cubic spline regression model

\section{Discussion}

In this Chinese population in Singapore, we found a strong dose-dependent association between higher serum FGF-21 levels and increased risk of incident type 2 diabetes in women but not in men, and the association was independent of liver enzymes and other diabetes risk factors. In addition, FGF-21 improved diabetes risk reclassification among women.

The positive association between FGF-21 and incident type 2 diabetes found in the current study is in accordance with previous studies [6-8]. A 5.4-year prospective cohort study among 1292 Chinese (73 diabetes cases) in Hong Kong reported an OR of 1.79 (95\% CI 1.22-2.64) for the risk of diabetes with per 1 unit increment in log FGF-21 levels, after adjusting for fasting glucose, insulin, TG, HDL-C and hs-CRP [7]. Later, an updated study in the same cohort focusing on diabetes prediction model reported that higher FGF-21 ( $\geq 178.2$ versus $<178.2 \mathrm{pg} / \mathrm{mL}$ ) was associated with an increased diabetes risk independent of other blood biomarkers (OR, 1.60; 95\% CI 1.18-2.16) [8]. So far, only one prospective study (440 Germans with 54 diabetes cases) has examined the impact of liver enzymes on the association between FGF-21 and diabetes [6]. The study found a positive association after adjusting for liver enzymes, although the risk estimate was not reported [6]. In the current study, we adjusted for liver enzymes individually and in combination with other blood biomarkers, and the results remained significant. We also stratified the analysis by liver enzymes and did not find significant effect modification.

A number of experimental evidence has shown that FGF-21 may involve in key etiological pathways leading to diabetes development such as regulation of lipid 
Table 3 Odds ratios (95\% confidence intervals) of incident type 2 diabetes by stratified analysis in the total samples, the Singapore Chinese Health Study ${ }^{a}$

\begin{tabular}{|c|c|c|c|}
\hline \multirow[t]{2}{*}{ Variables } & \multicolumn{2}{|c|}{ Per 1 log increment of FGF-21 } & \multirow[t]{2}{*}{$P$-interaction } \\
\hline & Cases/ controls & OR $(95 \% \mathrm{Cl})$ & \\
\hline \multicolumn{4}{|l|}{ Age (year) } \\
\hline$<60$ & $151 / 150$ & $1.35(1.04-1.76)$ & 0.39 \\
\hline$\geq 60$ & $100 / 101$ & $1.20(0.86-1.67)$ & \\
\hline \multicolumn{4}{|l|}{$\mathrm{BMI}, \mathrm{kg} / \mathrm{m}^{2}$} \\
\hline$<23$ & $117 / 117$ & $1.31(0.99-1.73)$ & 0.96 \\
\hline$\geq 23$ & $134 / 134$ & $1.26(0.94-1.70)$ & \\
\hline \multicolumn{4}{|l|}{ Physical activity } \\
\hline$<0.5 \mathrm{~h} /$ week & $84 / 148$ & $1.42(1.12-1.80)$ & 0.46 \\
\hline$\geq 0.5 \mathrm{~h} /$ week & 167/103 & $1.00(0.65-1.54)$ & \\
\hline \multicolumn{4}{|l|}{ Fasting status } \\
\hline Fasting & 196/194 & $1.25(0.99-1.57)$ & 0.64 \\
\hline Non-fasting & $55 / 57$ & $1.39(0.87-2.21)$ & \\
\hline \multicolumn{4}{|l|}{$\mathrm{Hs}-\mathrm{CRP}, \mathrm{mg} / \mathrm{L}$} \\
\hline$<1.4$ & $74 / 67$ & $1.38(1.03-1.85)$ & 0.93 \\
\hline$\geq 1.4$ & $177 / 184$ & $1.34(1.01-1.78)$ & \\
\hline \multicolumn{4}{|l|}{ GGT, IU/L } \\
\hline$<30$ & $102 / 140$ & $1.21(0.91-1.61)$ & 0.18 \\
\hline$\geq 30$ & $149 / 111$ & $1.37(1.00-1.89)$ & \\
\hline \multicolumn{4}{|l|}{$A L T, I U / L$} \\
\hline$<26$ & $96 / 147$ & $1.26(0.93-1.69)$ & 0.51 \\
\hline$\geq 26$ & 155/104 & $1.30(0.96-1.77)$ & \\
\hline \multicolumn{4}{|l|}{$\mathrm{TG}, \mathrm{mmol} / \mathrm{L}$} \\
\hline$<1.815$ & $91 / 140$ & $1.08(0.82-1.42)$ & 0.18 \\
\hline$\geq 1.815$ & $160 / 111$ & $1.42(1.02-1.97)$ & \\
\hline \multicolumn{4}{|l|}{$\mathrm{HDL}-\mathrm{C}, \mathrm{mmol} / \mathrm{L}$} \\
\hline$<0.99$ & $94 / 157$ & $1.12(0.80-1.56)$ & 0.87 \\
\hline$\geq 0.99$ & $157 / 94$ & $1.26(0.96-1.65)$ & \\
\hline
\end{tabular}

adds ratios were estimated after adjusting for adjusted for age at blood taken (continuous), smoking (never, ever smoker), alcohol intake (never, ever drinker), weekly moderate-to-vigorous activity $(<0.5, \geq 0.5$ hours/week), education level (primary school and below, secondary or above), history of hypertension (yes, no), fasting status (yes, no) and BMI (continuous), except for stratifying factors

homeostasis [22], inflammation [23], and development of NAFLD [9]. Increased levels of circulating free fatty acids induced liver FGF-21 secretion by a PPAR- $\alpha$ dependent pathway [24, 25], and raised FGF-21 lowered TG levels by speeding up lipoprotein catabolism in adipose tissues [26] and by regulating the expression of key genes involved in lipid metabolism [22]. In addition, inflammation in adipose tissue could suppress FGF-21 receptor $\beta$-klotho [27], which subsequently attenuated FGF-21 signaling and caused FGF-21 resistance in rodents [23]. However, the current analysis along with prior studies have shown that the association between
FGF-21 and diabetes was independent of lipids (TG, HDL-C), inflammatory marker (hs-CRP) and liver enzymes (GGT, ALT) [6-8]. In addition, FGF-21 could reduce glucose levels by inducing expression and secretion of adiponectin [28], and increasing glucose uptake in brown adipose tissues [22] and skeletal muscle [29]. In accordance with the mechanism, we observed a positive correlation between FGF-21 and adiponectin in the current analysis. However, since adiponectin is a consequence rather than a determinant of FGF-21, we did not include adiponectin in the multivariable model. Despite of FGF-21 being a risk factor for diabetes, recent animal study and clinical trials in humans have shown beneficial effects of exogenous FGF-21 on lipid profile, levels of adiponectin, fasting insulin and glucose in obese patients with diabetes [30-32], suggesting FGF-21 may also serve as a potential therapeutic agent for treating type 2 diabetes in humans.

In addition, we have observed sex-interaction in the association between FGF-21 and diabetes in this Chinese population. To the best of our knowledge, no prospective studies have examined the sex interaction with FGF-21 in relation to diabetes. However, three cross-sectional studies have reported significant interactions between FGF-21 and sex in the association with carotid atherosclerosis [10], lower extremity atherosclerotic disease [12] and bone mineral density [11]; in all three studies, significant positive correlations were only observed in women but not in men. The underlying mechanism for the observed sex heterogeneity may be due to different body fat distribution and sex hormone. Compared to men, women have greater accumulation of subcutaneous fat, and higher amount and activity of brown adipose tissue, which was hypothesized to impact whole-body energy metabolism, insulin resistance, and obesity-related diabetes [33]. In addition, animal studies have shown that estrogen increases hepatic production of FGF-21 [34] and enhance the activity of FGF21 in brown adipose tissue [35]. However, majority of the women were at postmenopausal status in the current study, and no significant interaction was observed with menopausal status. Therefore, whether estrogen played an important role in the association between FGF-21 and diabetes risk remains to be explored.

A prospective study in Hong Kong reported that addition of FGF-21 to a diabetes prediction model comprising of age, family history of type 2 diabetes, smoking, hypertension, BMI, dyslipidemia and fasting glucose showed statistically significant improvement in AUC from 0.797 to 0.819 , and its performance in diabetes prediction is comparable to the OGTT [8]. In contrast, another prospective cohort study in a German population reported that addition of FGF-21 to a base model including age, sex, BMI and time of follow-up did not yield statistically significant increment in AUC [6]. In the current study, we 
Table 4 Odds ratios (95\% confidence intervals) of type 2 diabetes by stratified analysis in men and women separately, the Singapore Chinese Health Study ${ }^{a}$

\begin{tabular}{lll}
\hline Variables & Per 1 log increment of FGF-21 & P-interaction \\
\cline { 2 - 2 } & Cases/ controls OR $(95 \% \mathrm{Cl})$ & \\
\hline
\end{tabular}

Men

Age $(\text { year })^{a}$$$
<60
$$$$
\geq 60
$$

$65 / 64$

$52 / 53$

$\mathrm{BMI}^{\mathrm{a}}, \mathrm{kg} / \mathrm{m}^{2}$

$$
<23
$$$$
33 / 67
$$$$
\geq 23
$$

$84 / 50$

Physical activity ${ }^{a}$

$$
<0.5 \mathrm{~h} / \text { week }
$$

$89 / 90$

$\geq 0.5 \mathrm{~h} /$ week

$28 / 27$

Fasting status ${ }^{a}$

$$
\text { Fasting }
$$

Non-fasting

$34 / 32$

$\mathrm{Hs}-\mathrm{CRP}^{\mathrm{a}}$, mg/L

$$
<1.4
$$

$\geq 1.4$

$\mathrm{GGT}^{\mathrm{a}}, \mathrm{IU} / \mathrm{L}$

$$
<30
$$$$
\geq 30
$$

$\mathrm{ALT}^{\mathrm{a}}, \mathrm{IU} / \mathrm{L}$

$$
<26
$$$$
\geq 26
$$

$\mathrm{TG}^{\mathrm{a}}, \mathrm{mmol} / \mathrm{L}$

$$
<1.815
$$$$
\geq 1.815
$$

$\mathrm{HDL}-\mathrm{C}^{\mathrm{a}}, \mathrm{mmol} / \mathrm{L}$

$\begin{array}{ll}<0.99 & 75 / 41 \\ \geq 0.99 & 42 / 76\end{array}$

\begin{tabular}{|c|c|c|c|}
\hline \multirow[t]{2}{*}{ Variables } & \multicolumn{2}{|c|}{ Per 1 log increment of FGF-21 } & \multirow[t]{2}{*}{ P-interaction } \\
\hline & Cases/ controls & OR $(95 \% \mathrm{Cl})$ & \\
\hline \multicolumn{4}{|l|}{ Menopausal status ${ }^{b}$} \\
\hline Premenopausal & $26 / 28$ & $1.14(0.61-2.12)$ & 0.50 \\
\hline Postmenopausal & $108 / 106$ & $1.57(1.15-2.14)$ & \\
\hline \multicolumn{4}{|l|}{$\mathrm{Hs}-\mathrm{CRP}^{\mathrm{b}}, \mathrm{mg} / \mathrm{L}$} \\
\hline$<1.4$ & $51 / 76$ & $1.49(0.99-2.24)$ & 0.67 \\
\hline$\geq 1.4$ & $83 / 58$ & $1.33(0.88-2.01)$ & \\
\hline \multicolumn{4}{|l|}{$\mathrm{GGT}^{\mathrm{b}}, \mathrm{IU} / \mathrm{L}$} \\
\hline$<30$ & $49 / 78$ & $1.34(0.96-1.86)$ & 0.10 \\
\hline$\geq 30$ & $85 / 56$ & $1.98(1.08-3.63)$ & \\
\hline \multicolumn{4}{|l|}{$\mathrm{ALT}^{\mathrm{b}}, \mathrm{IU} / \mathrm{L}$} \\
\hline$<20$ & $45 / 80$ & $1.36(0.95-1.95)$ & 0.25 \\
\hline$\geq 20$ & $89 / 54$ & $2.05(1.20-3.49)$ & \\
\hline \multicolumn{4}{|l|}{$\mathrm{TG}^{\mathrm{b}}, \mathrm{mmol} / \mathrm{L}$} \\
\hline$<1.66$ & $48 / 86$ & $1.42(0.97-2.07)$ & 0.52 \\
\hline$\geq 1.66$ & $86 / 48$ & $1.57(0.99-2.50)$ & \\
\hline \multicolumn{4}{|l|}{$\mathrm{HDL}-\mathrm{C}^{\mathrm{b}}, \mathrm{mmol} / \mathrm{L}$} \\
\hline$<1.23$ & $83 / 50$ & $1.16(0.59-2.32)$ & 0.69 \\
\hline$\geq 1.23$ & $51 / 84$ & $1.40(1.02-1.91)$ & \\
\hline
\end{tabular}

Women

$\begin{array}{llll}\begin{array}{l}\text { Age }(\text { year) } \\ <60\end{array} & 86 / 86 & 1.48(1.06-2.07) & 0.74 \\ \geq 60 & 48 / 48 & 1.44(0.86-2.41) & \\ \mathrm{BMI}^{\mathrm{b}}, \mathrm{kg} / \mathrm{m}^{2} & & & \\ <23 & 51 / 81 & 1.53(1.06-2.22) & 0.62 \\ \geq 23 & 83 / 53 & 1.43(0.95-2.14) & \\ \begin{array}{c}\text { Physical activity } \\ \text { b }\end{array} & & & \\ <0.5 \text { h/week } & 107 / 104 & 1.62(1.18-2.22) & 0.34 \\ \geq 0.5 \text { h/week } & 27 / 30 & 1.29(0.71-2.37) & \\ \text { Fasting status } & & & \\ \text { Fasting } & 94 / 99 & 1.43(1.06-1.92) & 0.56 \\ \text { Non-fasting } & 40 / 35 & 1.74(0.86-3.53) & \end{array}$

Table 4 Odds ratios (95\% confidence intervals) of type 2 diabetes by stratified analysis in men and women separately, the Singapore Chinese Health Study ${ }^{a}$ (Continued)

adds ratios were estimated after adjusting for adjusted for age at blood taken (continuous), smoking (never, ever smoker), alcohol intake (never, ever drinker), weekly moderate-to-vigorous activity $(<0.5, \geq 0.5$ hours/week), education level (primary school and below, secondary or above), history of hypertension (yes, no), fasting status (yes, no) and BMI (continuous), except for stratifying factors

${ }^{b}$ Odds ratio were adjusted for the abovementioned variables plus menopausal status

did not observe significant improvement in AUC change after adding FGF-21 into the models. Since AUC has the limitation of being relatively insensitive to model improvement [36], we further applied other statistical methods such as NRI and IDI that have shown to be useful in assessing the predictive utility of novel biomarkers [20, 21]. As a result, including FGF-21 in the model significantly improved NRI and IDI, and the best cut-off value $(123 \mathrm{pg} / \mathrm{mL})$ in the current study is much lower than the one identified in the Hong Kong study $(178.2 \mathrm{pg} / \mathrm{mL})$ [8], which may due to the fact that Hong Kong study used primarily fasting specimens, while the current study used mostly non-fasted samples. Since FGF-21 is not a routinely measured biomarker in the clinical practice yet, and the sensitivity $(75.7 \%)$ and specificity $(41.4 \%)$ of the best cut-off value were low in the current study, more studies are needed to validate the clinical potential of using FGF21 in diabetes prediction in daily practice.

The strength of the current study is the adjustment for well-established diabetes risk factors (including liver enzymes), and using comprehensive statistical methods 
Table 5 Summary statistics to assess binary FGF-21 in predicting incident type 2 diabetes risk among female participants, the Singapore Chinese Health Study ${ }^{a}$

\begin{tabular}{|c|c|c|c|c|}
\hline \multirow[t]{2}{*}{ Variable } & \multicolumn{4}{|l|}{ Multivariable models ${ }^{b}$} \\
\hline & Discrimination (AUC [95\% CI]) & Calibration (AIC) & NRI & $\mid \mathrm{DI}$ \\
\hline Base model $1^{c}$ & $0.622(0.555-0.689)$ & 156 & & \\
\hline Base model $1^{\mathrm{C}}+\mathrm{FGF}-21$ & $0.640(0.574-0.706)^{f}$ & 149 & $0.358(0.118-0.598)$ & $0.028(0.007-0.048)$ \\
\hline Base model $2^{d}$ & $0.764(0.707-0.820)$ & 130 & & \\
\hline Base model $2^{d}+$ FGF-21 & $0.768(0.712-0.824)^{f}$ & 128 & $0.358(0.118-0.598)$ & $0.013(0.001-0.025)$ \\
\hline Base model $3^{\mathrm{e}}$ & $0.792(0.739-0.845)$ & 135 & & \\
\hline Base model $3^{\mathrm{e}}+\mathrm{FGF}-21$ & $0.798(0.746-0.850)^{f}$ & 133 & $0.388(0.149-0.628)$ & $0.015(0.001-0.029)$ \\
\hline
\end{tabular}

${ }^{a}$ Binary FGF-21 was created using a cutoff point of 123 , with a sensitivity of $75.7 \%$, and specificity of $41.4 \%$

${ }^{\mathrm{b}}$ Multivariable model adjusted for all the variables included in the base model plus binary FGF-21 $(<123 \mathrm{vs} . \geq 123 \mathrm{pg} / \mathrm{mL})$

'Base model 1 included age (continuous) and BMI (continuous)

${ }^{\mathrm{d}}$ Base model 2 included variables in base model 1 plus smoking status (never, ever smoker), history of hypertension (yes, no), and levels of TG (mmol/L), $\mathrm{HDL}-\mathrm{C}(\mathrm{mmol} / \mathrm{L})$, and random glucose $(\mathrm{mmol} / \mathrm{L})$ (all in quartiles)

'Base model 3 included variables in base model 2 plus adiponectin $(\mu \mathrm{g} / \mathrm{mL})$ and hs-CRP (mg/L) (both in quartiles)

${ }^{f}$ Compared to the base model, the $P$-values for the differences of AUC after including FGF- 21 to the base model were 0.11 for base model $1,0.56$ for base model

2 and 0.41 for model 3

(AUC, NRI and IDI) to explore the predictive utility of FGF-21. Furthermore, we measured $\mathrm{HbA}_{1 \mathrm{c}}$ in the blood collected at baseline, and excluded those with $\mathrm{HbA}_{1 \mathrm{c}}>6.5 \%$ $(48 \mathrm{mmol} / \mathrm{mol})$ to avoid undiagnosed diabetes cases. However, there are some limitations as well. First, we measured FGF-21 only once, and this one-time measurement may not represent long-term profile. However, this could lead to non-differential misclassification of FGF-21 status and thus may underestimate the true association. In addition, some information such as height, weight, and history of hypertension were self-reported, and we did not have information on family history of type 2 diabetes at baseline, thus residual confounding may exist. Furthermore, more than $70 \%$ of blood samples were non-fasting, and therefore may influence the FGF-21 levels. However, we have adjusted for fasting status in the models and no significant interaction was found with fasting status, indicating that fasting status did not influence the associations in the present study. Moreover, the sample size in the stratified analyses was small and studies with larger sample size are needed to further explore whether the association could be modified by other variables. Finally, the current study used matched case-control study design, and although it is valid in studying associations, recent studies pointed out that it may introduce bias when studying predictive utility of biomarkers [37, 38].

\section{Conclusions}

In conclusion, we observed a strong, dose-dependent association between serum FGF-21 levels and increased risk of incident type 2 diabetes in Chinese women but not in men. Further researches are needed to validate the findings, to investigate the underlying biological mechanisms, and to examine the feasibility of targeting FGF-21 through pharmacological interventions to reduce the risk of diabetes in high-risk population.

\section{Additional file}

Additional file 1: Table S1. The pair-wise Pearson correlation coefficients between FGF-21, age, blood levels of liver enzymes, lipids, high-sensitivity Creactive protein and adiponectin among case and control participants, the Singapore Chinese Health Study. Table S2. Reclassification of type 2 diabetes cases and controls among female participants with no risk categories based on their serum concentrations of FGF-21, the Singapore Chinese Health Study. Figure S1. Flowchart of the Singapore Chinese Health Study. (DOCX $106 \mathrm{~kb}$ )

\section{Abbreviations}

AIC: Akaike information criteria; ALT: Alanine transaminase; AUC: Area under receiver-operating characteristic curve; BMl: Body mass index; $\mathrm{Cl}$ : Confidence interval; FGF-21: Fibroblast growth factor 21; GGT: Gamma-glutamyltransferase; HbA1c: Hemoglobin A1c; HDL-C: High-density lipoprotein cholesterol; Hs-CRP: High-sensitivity C-reactive protein; IDI: Integrated discrimination improvement; NAFLD: Nonalcoholic fatty liver disease; NRI: Net reclassification improvement; OGT: Oral glucose tolerance test; OR: Odds ratio; ROC: Receiver-operating characteristic; SD: Standard deviation; TC: Total cholesterol; TG: Triglycerides

\section{Acknowledgements}

We thank Siew-Hong Low of the National University of Singapore for supervising the fieldwork of the Singapore Chinese Health Study, and Renwei Wang for the maintenance of the cohort study database. We also thank the founding principal investigator of the Singapore Chinese Health Study, Mimi C. Yu.

\section{Funding}

This study was supported by the National Medical Research Council, Singapore (NMRC/CIRG/1354/2013) and National Institutes of Health, USA (RO1 CA144034 and UM1 CA182876). W-P Koh is supported by the National Medical Research Council, Singapore (NMRC/CSA/0055/2013).

Availability of data and materials

The datasets generated during and/or analyzed during the current study are available from the corresponding author on reasonable request.

\section{Authors' contributions}

AP and WPK conceived the study, interpreted the data, and critically revised the reports. YW analyzed and interpreted the data, drafted and critically revised the reports. JMY contributed to the acquisition of study materials and critically revised the reports. All authors revised and approved the final report. 


\section{Ethics approval and consent to participate}

The study protocol was approved by the Institutional Review Boards at the National University of Singapore and the University of Pittsburgh. Informed consent was provided and completed by each participant at the baseline interview.

\section{Consent for publication}

Not applicable.

\section{Competing interests}

The authors declare that they have no competing interests.

\section{Publisher's Note}

Springer Nature remains neutral with regard to jurisdictional claims in published maps and institutional affiliations.

\section{Author details \\ ${ }^{1}$ Saw Swee Hock School of Public Health, National University of Singapore and National University Health System, Singapore 117549, Singapore. ${ }^{2}$ Duke-NUS Medical School, Singapore 169857, Singapore. ${ }^{3}$ UPMC Hillman Cancer Center, University of Pittsburgh, Pittsburgh, PA 15232, USA. ${ }^{4}$ Department of Epidemiology, Graduate School of Public Health, University of Pittsburgh, Pittsburgh, PA 15261, USA. ${ }^{5}$ Department of Epidemiology and Biostatistics, Ministry of Education Key Laboratory of Environment and Health and State Key Laboratory of Environmental Health (incubation), School of Public Health, Tongji Medical College, Huazhong University of Science and Technology, Wuhan, Hubei Province 430030, China.}

Received: 13 June 2017 Accepted: 14 September 2017

Published online: 30 September 2017

\section{References}

1. Woo YC, Xu A, Wang Y, Lam KS. Fibroblast growth factor 21 as an emerging metabolic regulator: clinical perspectives. Clin Endocrinol. 2013;78:489-96.

2. Kharitonenkov A, Shiyanova TL, Koester A, Ford AM, Micanovic R, Galbreath EJ, et al. FGF-21 as a novel metabolic regulator. J Clin Invest. 2005;115:1627-35.

3. Kharitonenkov A, DiMarchi R. FGF21 revolutions: recent advances illuminating FGF21 biology and medicinal properties. Trends Endocrinol Metab. 2015;26:608-17.

4. Zhang X, Yeung DC, Karpisek M, Stejskal D, Zhou ZG, Liu F, et al. Serum FGF21 levels are increased in obesity and are independently associated with the metabolic syndrome in humans. Diabetes. 2008:57:1246-53.

5. Chavez AO, Molina-Carrion M, Abdul-Ghani MA, Folli F, Defronzo RA, Tripathy D. Circulating fibroblast growth factor-21 is elevated in impaired glucose tolerance and type 2 diabetes and correlates with muscle and hepatic insulin resistance. Diabetes Care. 2009:32:1542-6.

6. Bobbert T, Schwarz F, Fischer-Rosinsky A, Pfeiffer AF, Mohlig M, Mai K, et al. Fibroblast growth factor 21 predicts the metabolic syndrome and type 2 diabetes in Caucasians. Diabetes Care. 2013;36:145-9.

7. Chen C, Cheung BM, Tso AW, Wang Y, Law LS, Ong KL, et al. High plasma level of fibroblast growth factor 21 is an independent predictor of type 2 diabetes: a 5.4-year population-based prospective study in Chinese subjects. Diabetes Care 2011:34:2113-5.

8. Woo YC, Lee $\mathrm{CH}$, Fong $\mathrm{CH}$, Xu A, Tso AW, Cheung BM, et al. Serum fibroblast growth factor 21 is a superior biomarker to other adipokines in predicting incident diabetes. Clin Endocrinol. 2017;86:37-43.

9. Li H, Fang Q, Gao F, Fan J, Zhou J, Wang X, et al. Fibroblast growth factor 21 levels are increased in nonalcoholic fatty liver disease patients and are correlated with hepatic triglyceride. J Hepatol. 2010:53:934-40.

10. Chow WS, Xu A, Woo YC, Tso AW, Cheung SC, Fong CH, et al. Serum fibroblast growth factor-21 levels are associated with carotid atherosclerosis independent of established cardiovascular risk factors. Arterioscler Thromb Vasc Biol. 2013:33:2454-9.

11. Lee $P$, Linderman J, Smith S, Brychta RJ, Perron R, Idelson C, et al. Fibroblast growth factor 21 (FGF21) and bone: is there a relationship in humans? Osteoporos Int. 2013:24:3053-7.

12. Zhang $X, H u$ Y, Zeng H, Li L, Zhao J, Zhao J, et al. Serum fibroblast growth factor 21 levels is associated with lower extremity atherosclerotic disease in Chinese female diabetic patients. Cardiovasc Diabetol. 2015;14:32.

13. Unger RH. Minireview: weapons of lean body mass destruction: the role of ectopic lipids in the metabolic syndrome. Endocrinology. 2003;144:5159-65.
14. Wang YL, Koh WP, Yuan JM, Pan A. Association between liver enzymes and incident type 2 diabetes in Singapore Chinese men and women. BMJ Open Diabetes Res Care. 2016;4:e000296.

15. Hankin JH, Stram DO, Arakawa K, Park S, Low SH, Lee HP, et al. Singapore Chinese health study: development, validation, and calibration of the quantitative food frequency questionnaire. Nutr Cancer. 2001;39:187-95.

16. Odegaard AO, Pereira MA, Koh WP, Arakawa K, Lee HP, Yu MC. Coffee, tea, and incident type 2 diabetes: the Singapore Chinese health study. Am J Clin Nutr. 2008;88:979-85.

17. Li X, Fan X, Ren F, Zhang Y, Shen C, Ren G, et al. Serum FGF21 levels are increased in newly diagnosed type 2 diabetes with nonalcoholic fatty liver disease and associated with hsCRP levels independently. Diabetes Res Clin Pract. 2011;93:10-6.

18. Youden WJ. Index for rating diagnostic tests. Cancer. 1950;3:32-5.

19. DeLong ER, DeLong DM, Clarke-Pearson DL. Comparing the areas under two or more correlated receiver operating characteristic curves: a nonparametric approach. Biometrics. 1988;44:837-45.

20. Pencina MJ, D'Agostino RB Sr, D'Agostino RB Jr, Vasan RS. Evaluating the added predictive ability of a new marker: from area under the ROC curve to reclassification and beyond. Stat Med. 2008;27:157-72.

21. Pencina MJ, D'Agostino RB Sr, Steyerberg EW. Extensions of net reclassification improvement calculations to measure usefulness of new biomarkers. Stat Med. 2011;30:11-21.

22. Emanuelli B, Vienberg SG, Smyth G, Cheng C, Stanford Kl, Arumugam M, et al. Interplay between FGF21 and insulin action in the liver regulates metabolism. J Clin Invest. 2015;125:458.

23. Diaz-Delfin J, Hondares E, Iglesias R, Giralt M, Caelles C, Villarroya F. TNF-alpha represses beta-Klotho expression and impairs FGF21 action in adipose cells: involvement of JNK1 in the FGF21 pathway. Endocrinology. 2012;153:4238-45.

24. Badman MK, Pissios P, Kennedy AR, Koukos G, Flier JS, Maratos-Flier E. Hepatic fibroblast growth factor 21 is regulated by PPARalpha and is a key mediator of hepatic lipid metabolism in ketotic states. Cell Metab. 2007;5:426-37.

25. Mai K, Andres J, Biedasek K, Weicht J, Bobbert T, Sabath M, et al. Free fatty acids link metabolism and regulation of the insulin-sensitizing fibroblast growth factor-21. Diabetes. 2009;58:1532-8.

26. Schlein C, Talukdar S, Heine M, Fischer AW, Krott LM, Nilsson SK, et al. FGF21 lowers plasma triglycerides by accelerating lipoprotein catabolism in white and Brown adipose tissues. Cell Metab. 2016;23:441-53.

27. Kharitonenkov A, Dunbar JD, Bina HA, Bright S, Moyers JS, Zhang C, et al. FGF-21/FGF-21 receptor interaction and activation is determined by betaKlotho. J Cell Physiol. 2008;215:1-7.

28. Lin Z, Tian H, Lam KS, Lin S, Hoo RC, Konishi M, et al. Adiponectin mediates the metabolic effects of FGF21 on glucose homeostasis and insulin sensitivity in mice. Cell Metab. 2013;17:779-89.

29. Mashili FL, Austin RL, Deshmukh AS, Fritz T, Caidahl K, Bergdahl K, et al. Direct effects of FGF21 on glucose uptake in human skeletal muscle: implications for type 2 diabetes and obesity. Diabetes Metab Res Rev. 2011; 27:286-97.

30. Hale C, Chen MM, Stanislaus S, Chinookoswong N, Hager T, Wang M, et al. Lack of overt FGF21 resistance in two mouse models of obesity and insulin resistance. Endocrinology. 2012;153:69-80.

31. Gaich G, Chien JY, Fu H, Glass LC, Deeg MA, Holland WL, et al. The effects of LY2405319, an FGF21 analog, in obese human subjects with type 2 diabetes. Cell Metab. 2013;18:333-40.

32. Talukdar S, Zhou Y, Li D, Rossulek M, Dong J, Somayaji V, et al. A longacting FGF21 molecule, PF-05231023, decreases body weight and improves lipid profile in non-human primates and type 2 diabetic subjects. Cell Metab. 2016;23:427-40.

33. Kautzky-Willer A, Harreiter J, Pacini G. Sex and gender differences in risk, Pathophysiology and complications of type 2 diabetes mellitus. Endocr Rev. 2016:37:278-316.

34. Kim JH, Meyers MS, Khuder SS, Abdallah SL, Muturi HT, Russo L, et al. Tissueselective estrogen complexes with bazedoxifene prevent metabolic dysfunction in female mice. Mol Metab. 2014;3:177-90.

35. Grefhorst A, van den Beukel JC, van Houten EL, Steenbergen J, Visser JA, Themmen AP. Estrogens increase expression of bone morphogenetic protein 8b in brown adipose tissue of mice. Biol Sex Differ. 2015:6:7.

36. Pepe MS, Janes $H$, Longton $G$, Leisenring W, Newcomb P. Limitations of the odds ratio in gauging the performance of a diagnostic, prognostic, or screening marker. Am J Epidemiol. 2004;159:882-90. 
37. Pepe MS, Fan J, Seymour CW, Li C, Huang Y, Feng Z. Biases introduced by choosing controls to match risk factors of cases in biomarker research. Clin Chem. 2012;58:1242-51.

38. Ganna A, Reilly M, de Faire U, Pedersen N, Magnusson P, Ingelsson E. Risk prediction measures for case-cohort and nested case-control designs: an application to cardiovascular disease. Am J Epidemiol. 2012;175:715-24.

Submit your next manuscript to BioMed Central and we will help you at every step:

- We accept pre-submission inquiries

- Our selector tool helps you to find the most relevant journal

- We provide round the clock customer support

- Convenient online submission

- Thorough peer review

- Inclusion in PubMed and all major indexing services

- Maximum visibility for your research

Submit your manuscript at www.biomedcentral.com/submit 